\title{
Feasibility study of LTE signal as a new illuminators of opportunity for passive radar applications
}

\begin{abstract}
Recently, there has been an evolution of mobile networks towards the fourth generation radio wireless communications (4G) as LTE (Long Term Evolution). In this paper, the feasibility of using LTE-based passive radar is investigated to take advantage of using LTE signal as illuminator of opportunity for moving object monitoring. An analysis of ambiguity function is done on a typical LTE waveform to assess the Doppler and range characteristics. The initial results and analysis show that LTE signal range and Doppler resolutions of $7.5 \mathrm{~m}$ and $0.11 \mathrm{~m} / \mathrm{s}$ can be achieved, respectively.
\end{abstract}

Keyword: LTE; Range resolution; Doppler resolution; Ambiguity function; Illuminators of opportunity; Passive radar 